\title{
AN ASYMPTOTIC PROPERTY OF THE ROOTS OF POLYNOMIALS
}

\author{
HERMANN FLASCHKA ${ }^{1}$
}

ABSTRACT. It is shown that if the imaginary parts of the roots $\lambda_{j}(s)$ of a polynomial $P(\lambda, s), s \in R^{n}$, are unbounded for large $|s|$, then they are in fact unbounded along a one-parameter algebraic curve $s=s(R)$. The result may be used to reduce certain questions about polynomials in several variables to an essentially one-dimensional form; this is illustrated by an application to hyperbolic poly. nomials.

For a constant-coefficient partial differential equation

$$
P\left(-i \partial / \partial t,-i \partial / \partial x_{1}, \cdots,-i \partial / \partial x_{N}\right) u=0
$$

the correctness of the Cauchy problem, in the sense of Petrowsky, is a purely algebraic question: do the roots $\lambda_{j}(s)$ of $P(\lambda, s)=0$ satisfy

$$
\left|\operatorname{Im} \lambda_{j}(s)\right| \leqq \text { const }
$$

for all $s$ in $R^{N}$ ? If $N=1$, the roots can be expanded in a Puiseux series [1]

$$
\lambda_{j}(s)=\sum_{k=-p}^{\infty} a_{k} s^{q_{k}}
$$

where the exponents $q_{k}$ tend to $-\infty$ as $k \rightarrow \infty$. Under the assumption that (2) is violated, the function $u_{s}(t, x)=\exp i\left(\lambda_{j}(s) t+s x\right)$ is a solution of (1) which, either for $t<0$ or for $t>0$, grows exponentially in the parameter $s$, while its values and those of its derivatives at $t=0$ depend only polynomially on $s$. Incorrectness of the Cauchy problem is essentially a consequence of this domination of the Cauchy data by the solution. A generalized Puiseux series has been used by Strang [2] to establish necessary conditions for correctness of equations with variable coefficients. ${ }^{2}$ This technique does not extend to the case $N>1$, however, because the Puiseux expansion is then no longer available. If condition (2) should happen to be violated along

Received by the editors April 16, 1970.

A MS 1969 subject classifications. Primary 1230, 3553; Secondary 3537.

Key words and phrases. Roots of polynomials, Seidenberg-Tarski theorem, hyperbolic polynomials.

1 NSF Graduate Fellow.

2 (See also H. Flaschka and G. Strang, The correctness of the Cauchy problem, to appear in Advances in Math.) 
a ray $s=\left(a_{1} r, \cdots, a_{N} r\right)=a r$, with $a \in R^{N}$ fixed,

$$
\operatorname{Im} \lambda_{j}(\text { ar }) \rightarrow \infty \quad \text { as } r \rightarrow \infty \text {, }
$$

then correctness could still be established by investigation of a Puiseux expansion in powers of $r$. Unfortunately, there is an example due to Petrowsky,

$$
P(\lambda, s)=\lambda^{3}-s_{1} \lambda^{2}-s_{2}^{2} \lambda+i s_{2} \lambda-i s_{1} s_{2}
$$

which shows that, although $\operatorname{Im} \lambda_{j}$ (ar) may be bounded along each ray $s=a r$, it may fail to be bounded uniformly over all rays and may become infinite on more general curves through $s$-space; in this instance, $\operatorname{Im} \lambda_{j}\left(r^{2}, r\right) \rightarrow \infty$. The example is, in fact, general. The purpose of this note is to show that if (2) is violated, then violation must occur along an algebraic curve $s=s(R)$ (Theorem 1). Thus there is still a possibility of expanding the $\lambda_{j}$ in a Puiseux series in the parameter $R$, and proceeding with the incorrectness construction as for the case $N=1$.

The proof of Theorem 1 relies heavily on the Seidenberg-Tarski theorem (see [3] and below), and, as might be expected, can be modified easily to yield information about the manner in which more general algebraic inequalities are violated. This more inclusive statement is given in Theorem 2, followed by a brief sketch of a situation in which it may be of use.

Theorem 1 was conjectured by Professor G. Strang in the course of a study of the correctness of the Cauchy problem [2]; I would like to thank him for numerous helpful suggestions.

2. Let $P(\lambda, s)=\sum_{k=0}^{m} p_{k}(s) \lambda^{k}$, where $p_{k}(s)$ is a polynomial of degree $m-k$. We define

$$
\Lambda(s)=\max _{j} \operatorname{Im} \lambda_{j}(s) \text { and } \bar{\Lambda}(r)=\sup _{|s|=r} \Lambda(s) .
$$

It can be shown (see [3]) that for sufficiently large $r, \bar{\Lambda}(r)$ is an algebraic function: $\bar{\Lambda}(r)=A r^{h}+\cdots$. We assume that (2) is violated, so that necessarily $h>0$; let us also suppose that $A>0$.

TheOREM 1. Let $\epsilon$ satisfying $0<\epsilon<A$ be given. There exist polynomials $G_{1}, \cdots, G_{N}$ in two variables, and functions $s_{j}(R), j=1, \cdots$, $N$, satisfying

$$
G_{j}\left(s_{j}(R), R\right)=0, \quad j=1, \cdots, N,
$$

for sufficiently large $R$, such that 


$$
\Lambda(s(R))>(A-\epsilon)|s(R)|^{h},
$$

for large $R .\left(\right.$ Here $s(R)=\left(s_{1}(R), \cdots, s_{N}(R)\right)$.)

Proof. Write $\lambda=\mu_{1}+i \mu_{2}$, and $P(\lambda, s)=P_{1}\left(\mu_{1}, \mu_{2}, s\right)+i P_{2}\left(\mu_{1}, \mu_{2}, s\right)$, with $P_{1}$ and $P_{2}$ real polynomials. Then by assumption, the following system of (real) polynomial equalities and inequalities will have real solutions for large $R$ :

$$
P_{1}\left(\mu_{1}, \mu_{2}, s\right)=0, \quad P_{2}\left(\mu_{1}, \mu_{2}, s\right)=0,
$$

$$
|s|^{2}=R^{2 q}, \quad \mu_{2}>(A-\epsilon) R^{p}
$$

we have set $r=R^{q}$, where $p$ and $q$ are integers with $h=p / q$. According to the Seidenberg-Tarski theorem, there exists a set $K_{1}, \cdots, K_{d}$ of systems of polynomial equations and inequalities in the two variables $s_{1}$ and $R$, such that a given pair $\left(s_{1}^{\circ}, R^{\circ}\right)$ may be extended to a solution $s_{1}^{\circ}, s_{2}, \cdots, s_{N}, \mu_{1}, \mu_{2}, R^{\circ}$ of $\left(\mathrm{S}_{1}\right)$ if and only if $\left(s_{1}^{\circ}, R^{\circ}\right)$ satisfies at least one of the systems $K_{j}$.

Concerning these $K_{j}$, we note first that at least one of them must have solutions $R, s_{1}(R)$ for infinitely many $R, R \rightarrow \infty$, because $\left(\mathrm{S}_{1}\right)$ has solutions for large $R$. In addition, not all the systems $K_{j}$ may contain an equation. Indeed, if this were the case, then for each $R^{\circ}$ the value of $s_{1}$ in any solution $s_{1}, \cdots, R^{\circ}$ of $\left(\mathrm{S}_{1}\right)$ would be restricted to a finite set. However, because of the continuous dependence of the roots of $P(\lambda, s)=0$ on $s$, if $s_{1}^{\circ}, \cdots, R^{\circ}$ is a solution of $\left(\mathrm{S}_{1}\right)$, then a small variation of $s_{1}$ may be compensated for by variations of $s_{2}, \cdots, \mu_{2}$, without violation of any of the conditions $\left(\mathrm{S}_{1}\right)$. There are, therefore, infinitely many $s_{1}$ for which the pair $\left(s_{1}, R^{\circ}\right)$ may be extended to a solution of $\left(\mathrm{S}_{1}\right)$. Finally, it is clear that at least one of the $K_{j}$ which have solutions $s_{1}, R$ for infinitely many $R, R \rightarrow \infty$, must consist of inequalities only; we take this system to be $K_{1}$.

Suppose $K_{1}$ has the form

$$
f_{i}\left(s_{1}, R\right) \geqq 0 \quad(\text { or }>0), \quad i=1, \cdots, e .
$$

The solution curves of the equations $f_{i}\left(s_{1}, R\right)=0$ divide the $\left(s_{1}, R\right)$ plane (outside of a bounded region) into finitely many strips; because of the assumption on $K_{1}$, there will be at least one such strip, say $T$, in the interior of which all $f_{i}$ are positive. Suppose $T$ is bounded (again, for $R$ large) by the algebraic curves $s_{1}=g_{1}(R), s_{2}=g_{2}(R)$; ultimately, the curve $s_{1}=\frac{1}{2}\left(g_{1}(R)+g_{2}(R)\right)=a_{1} R^{k_{1}}+\cdots$ will lie in $T$, and, provided that enough terms are included, so will the partial Puiseux expansion 


$$
s_{1}=h(R)=a_{1} R^{k_{1}}+\cdots+a_{f} R^{k j} .
$$

Put $f_{i}(h(R), R)=Q_{i}(R)$. A substitution $R^{\prime}=R^{1 / q^{\prime}}$, with $q^{\prime}$ an integer, will convert the $Q_{i}^{\prime}$ into expressions involving only powers and reciprocals of $R^{\prime}: Q_{i}(R)=Q_{i}^{\prime}\left(R^{\prime}\right)$. Then multiplication of

$$
\left(f_{1}\left(s_{1}, R^{\prime q^{\prime}}\right)-Q_{1}{ }^{\prime}\left(R^{\prime}\right)\right)^{2}+\cdots+\left(f_{e}\left(s_{1}, R^{\prime q^{\prime}}\right)-Q_{e}^{\prime}\left(R^{\prime}\right)\right)^{2}
$$

by a sufficiently high power of $R^{\prime}$ will result in a polynomial, $G_{1}\left(s_{1}, R^{\prime}\right)$, with the following property:

For sufficiently large $R^{\prime}$, if $s_{1}^{\circ}, R^{\circ \prime}$ satisfies $G_{1}\left(s_{1}^{\circ}, R^{\circ \prime}\right)=0$, the pair $s_{1}^{\circ}, R^{\circ}$ (with $R^{\circ}=R^{\circ / q^{\prime}}$ ) may be extended to a solution of $\left(\mathrm{S}_{1}\right)$. Indeed, from $G_{1}\left(s_{1}^{\circ}, R^{\circ \prime}\right)=0$ follows, for each $i=1, \cdots, e$, that $f_{i}\left(s_{1}^{\circ}, R^{\circ \prime q^{\prime}}\right)$ $=Q_{i}^{\prime}\left(R^{\circ \prime}\right)$. Hence $f_{i}\left(s_{1}^{\circ}, R^{\circ}\right)=Q_{i}\left(R^{\circ}\right)=f_{i}\left(h\left(R^{\circ}\right), R^{\circ}\right)>0$, so that $s_{1}^{\circ}, R^{\circ}$ satisfy the system $K_{1}$.

In a similar fashion, one now obtains a polynomial $G_{2}\left(s_{2}, R^{\prime \prime}\right)$, where $R^{\prime \prime}=\left(R^{\prime}\right)^{1 / q^{\prime \prime}}$, with the property:

For sufficiently large $R^{\prime \prime}$, if $\left(s_{2}^{\circ}, R^{\circ \prime \prime}\right)$ satisfies $G_{2}\left(s_{2}^{\circ}, R^{\circ \prime \prime}\right)=0$, then $s_{2}^{\circ}, R^{\circ \prime}$ (with $R^{\circ \prime}=\left(R^{\circ \prime \prime}\right)^{1 / q^{\prime \prime}}$ ) may be extended to a solution of

$$
P_{1}\left(\mu_{1}, \mu_{2}, s\right)=0, \quad P_{2}\left(\mu_{1}, \mu_{2}, s\right)=0,
$$

$$
|s|^{2}=\left(R^{\prime}\right)^{2 q q^{\prime}}, \quad \mu_{2}>(A-\epsilon)\left(R^{\prime}\right)^{p q^{\prime}}, \quad G_{1}\left(s_{1}, R^{\prime}\right)=0 .
$$

It must be noted that $\left(\mathrm{S}_{2}\right)$ does indeed have solutions for large $R^{\prime}$; this follows from the defining property of $G_{1}$.

After $N-1$ steps, one obtains polynomials $G_{j}\left(s_{j}, \bar{R}\right), j=1, \cdots$, $N-1$, in $s_{j}$ and a parameter $\bar{R}$ which is related to $r$ by $r=\bar{R} \bar{q}, \bar{q}$ integer, such that the system

$$
\begin{aligned}
& P_{1}\left(\mu_{1}, \mu_{2}, s\right)=0, \quad P_{2}\left(\mu_{1}, \mu_{2}, s\right)=0, \\
& |s|^{2}=\bar{R}^{2 \bar{q} q}, \quad \mu_{2}>(A-\epsilon) \bar{R}^{p \bar{q}}, \\
& G_{j}\left(s_{j}, \bar{R}\right)=0, \quad j=1, \cdots, N-1
\end{aligned}
$$

has solutions for infinitely many $\bar{R}, \bar{R} \rightarrow \infty$.

Now there are only finitely many piecewise algebraic curves $s_{1}$ $=s_{1}(\bar{R}), \cdots, s_{N}=s_{N}(\bar{R})$ which satisfy the conditions $G_{j}\left(s_{j}(\bar{R}), \bar{R}\right)$ $=0,|s|^{2}=\bar{R}^{2 \bar{q}}$. It follows then that there is a curve $s=s(\bar{R})$, algebraic for sufficiently large $\bar{R}$, along which we eventually have

$$
\mu_{2}>(A-\epsilon) \bar{R}^{p \bar{q}} \text {. }
$$

This proves the theorem.

Trivial modifications in the proof of Theorem 1 serve to establish 
Theorem 2. Let $s \in R^{N}, t \in R^{M}$, and let $K$ be a system of real polynomial equations and inequalities in $s$ and $t$. Suppose further that $\Lambda(s)$ is a function having the property that if $t_{1}$ is replaced by $\Lambda(s)$ in each equation or inequality in $K$, then the resulting system has real solutions $t_{2}, \cdots, t_{M}$ for all $s$. If, in addition, $\bar{\Lambda}(r)=\sup _{|S|=r} \Lambda(s)$ is algebraic for large $r$, and if $\bar{\Lambda}(r)=A r^{h}+\cdots$ with $h>0$, then there is an algebraic curve $s=s(R)$, defined by polynomial equations (4), such that the inequality (5) is again satisfied.

3. In conclusion we outline another situation in which Theorem 2 can be applied. Consider a polynomial $P(\lambda, s)=\sum_{\boldsymbol{t}=0}^{m} Q_{k}(\lambda, s)$, expressed as a sum of polynomials $Q_{k}$ homogeneous of degree $k$, and suppose that $Q_{m}(1,0)=1$. Let the distinct roots $\lambda=\mu_{j}(s)$ of $Q_{m}(\lambda, s)$ $=0$ be real and of constant multiplicities $\alpha_{j}, j=1, \cdots, r$. Defining $\partial_{j}=\lambda-\mu_{j}(s)$, we write $Q_{m}(\lambda, s)=\partial_{1}^{\alpha_{1}} \cdots \partial_{r}^{\alpha_{r}}$, or simply $Q_{m}=\partial^{\alpha}$, with the multi-index notation $\alpha=\left(\alpha_{1}, \cdots, \alpha_{r}\right)$. Finally, for a general polynomial $R(t), t \in R^{M}$, define

$$
\tilde{R}(t)=\left(\sum_{\beta}\left|(\partial / \partial t)^{\beta} R(t)\right|^{2}\right)^{1 / 2},
$$

the summation extending over all $\beta=\left(\beta_{1}, \cdots, \beta_{M}\right)$.

It is known from work of Hörmander [4] and Svensson [5], that the roots of $P(\lambda, s)$ will satisfy (2), i.e., $P$ will be hyperbolic, if and only if

$$
\left|Q_{k}(\lambda, s)\right| \leqq \text { const } \tilde{P}(\lambda, s)
$$

for all $k=0, \cdots, m$ and $(\lambda, s) \in R^{N+1}$. We now assert that this is equivalent to

$Q_{k}$ can be written as a linear combination of products $\partial^{\beta}$, with $\beta \leqq \alpha$ and $|\beta|=k$, and with coefficients which are bounded functions of $s$.

Proof. Let $(\lambda(R), s(R))$ be an algebraic curve. Put $f_{j}(R)=\lambda(R)$ $-\mu_{j}(s(R))$, and suppose that $f_{1}, \cdots, f_{d}$ are the only $f_{j}$ which tend to zero as $R \rightarrow \infty$. If we now put $\alpha_{1}+\cdots+\alpha_{d}=a$ and $\bar{\alpha}$ $=\left(0, \cdots, 0, \alpha_{d+1}, \cdots, \alpha_{r}\right)$, then it is clear that of all the products $f^{\beta}=f_{1}^{\beta_{1}} \cdots f_{r}^{\beta_{r}}, \beta \leqq \alpha$, the one having the most rapid growth with $R$ is $f^{\bar{\alpha}}$. Because

$$
\frac{\partial^{a}}{\partial \lambda^{a}} Q_{m}(\lambda, s)=\text { const } \partial^{\bar{\alpha}}+\text { terms involving } \partial_{1}, \cdots, \partial_{d},
$$

it follows that 


$$
\frac{\partial^{a}}{\partial \lambda^{a}} Q_{m}(\lambda(R), s(R))=\text { const } f^{\bar{\alpha}}+o\left(\overline{\alpha^{\bar{\alpha}}}\right), \quad R \rightarrow \infty .
$$

This implies that for any $\beta, \beta \leqq \alpha$,

$$
\left|f^{\beta}\right| \leqq \text { const } \tilde{Q}_{m}(\lambda(R), s(R)),
$$

so that if $Q_{k}$ has the form prescribed in (8), then the domination inequality (7) is satisfied along each algebraic curve and hence, by Theorem 2, uniformly in $\lambda$ and $s$. Condition (8) is therefore sufficient for hyperbolicity.

The necessity of (8) was proved by A. Lax [6] and Yamaguti [7], as was, in fact, the sufficiency; we present our alternate proof partly to illustrate the main theorem, and partly because the same technique can be employed when the roots $\mu_{j}$ have variable multiplicity to produce a description of the lower-order terms of a hyperbolic operator not yet contained in the literature. This extension, as well as applications to equations with variable coefficients, will be discussed in another paper.

\section{BIBLIOGRAPHY}

1. R. J. Walker, Algebraic curves, Dover, New York, 1962. MR 26 \#2438.

2. G. Strang, On multiple characteristics and the Levi-Lax conditions for hyperbolicity, Arch. Rational Mech. Anal. 33 (1969), 358-373. MR 39 \#4509.

3. A. Friedman, Generalized functions and partial differential equations, PrenticeHall, Englewood Cliffs, N. J., 1963. MR 29 \#2672.

4. L. Hörmander, Linear partial differential operators, Die Grundlehren der math. Wissenschaften, Band 116, Academic Press, New York and Springer-Verlag, Berlin, 1963. MR 28 \#4221.

5. L. Svensson, Necessary and sufficient conditions for the hyperbolicity of polynomials with hyperbolic principal part, Ark. Mat. 8 (1969), 145-162.

6. A. Lax, On Cauchy's problem for partial differential equations with multiple characteristics, Comm. Pure Appl. Math 9 (1956), 135-169. MR 18, 397.

7. M. Yamaguti, Le problème de Cauchy et les opérateurs d'intégrale singulière, Mem. Coll. Sci. Univ. Kyoto Ser. A Math. 32 (1959), 121-151. MR 22 \#146.

Massachusetts Institute of Technology, Cambridge, Massachusetts 02139 